\title{
Modulation of meiotic homologous recombination by DNA helicases
}

\author{
Alexander Lorenz \\ Institute of Medic al Sciences, University of Aberdeen, Foresterhill, Aberdeen AB25 2ד, UK \\ Correspondence to Alexander Lorenz (Phone: +44 1224 437323, Fax: +441224 437465, e-mail: a.lorenz@abdn.ac.uk)
}

Yeast (2016) DOI:10.1002/yea.3227

This is the peer reviewed version of the following article: Lorenz A (2016) Modulation of meiotic homologous recombination by DNA helicases. - Yeast, which has been published in final form at [http://dx.doi.org/10.1002/yea.3227]. This article may be used for noncommercial purposes in accordance with Wiley Terms and Conditions for Self-Archiving.

\begin{abstract}
DNA helicases are ATP-driven motor proteins which translocate along DNA capable of dismantling DNA-DNA interactions and/or removing proteins bound to DNA. These biochemical capabilities make DNA helicases main regulators of crucial DNA metabolic processes, including DNA replication, DNA repair, and genetic recombination. This budding topic will focus on reviewing the function of DNA helicases important for homologous recombination during meiosis, and discuss recent advances in how these modulators of meiotic recombination are themselves regulated. The emphasis is placed on work in the two model yeasts, Saccharomyces cerevisiae and Schizosaccharomyces pombe, which has vastly expanded our understanding of meiotic homologous recombination, a process whose correct execution is instrumental for healthy gamete formation, and thus functioning sexual reproduction.
\end{abstract}

Key words DNA helicases, homologous recombination, meiosis, crossover, template choice

\section{Introduction}

Meiosis is a specialized type of cell division employed to halve the genetic material of a diploid parental cell to produce haploid gametes (meiospores in fungi and plants, egg and sperm cells in other eukaryotes) (reviewed in [Hunter 2015]). To achieve this, one round of DNA replication is followed by two rounds of chromosome segregation. Remarkably, correct segregation of chromosomes in meiosis requires the deliberate formation of DNA double-strand breaks (DSBs) followed by repair through a process called meiotic (homologous) recombination. Recombination establishes physical connections (chiasmata) between maternal and paternal chromosomes that guide their proper segregation, and promotes reciprocal exchange of maternal and paternal genetic information (crossovers). The meiotic recombination process Lorenz (2016) combined with independent assortment of maternal and paternal chromosomes between gametes thus sustains genetic diversity and provides a driving force for evolution.

During meiosis homologous recombination is initiated by a programmed DSB made by the transesterase Spo11 (a.k.a. Rec12 in Schizosaccharomyces pombe) supported by a host of cofactors (Fig. 1A) (reviewed in [Lam and Keeney 2015]). The DSB ends are processed to remove Spo11 and resected to expose 3' single-stranded DNA tails (Fig. 1A). After loading of Dmc1 and/or Rad51 to form a nucleoprotein filament one of these 3' tails searches for a homologous repair template, during meiosis interactions with the homologous chromosome (homologue) in an allelic position are provided for in both yeasts, but favoured over interactions with the identical template on the sister chromatid only in Saccharomyces cerevisiae (Fig. 1A) [Schwacha and Kleckner 1997; Cromie et al. 2006; Hyppa and Smith 2010]. The homologous repair template is then invaded by the DSB end to form a D-loop (displacement loop), which can be extended by DNA synthesis (Fig. 1A). Dloops can be dismantled by DNA helicases. If dismantled in unextended form the nucleoprotein filament will again search for a homologous template. If in contrast it was already extended then the nucleoprotein filament can potentially be fed into the synthesis-dependent strand annealing (SDSA) pathway (Fig. 1A). By capturing the second end of the DSB an extended D-loop can also progress to form single or double Holliday junctions (HJs) (Figs. 1B, C). Single HJs are the predominant meiotic recombination intermediate in $S z$. pombe, whereas in S. cerevisiae mostly double HJs have been observed [Schwacha and Kleckner 1995; Cromie et al. 2006]. D-loops and HJs can be processed by Yeast DOI:10.1002/ yea.3227 
endonucleolytic cleavage resulting in crossovers or non-crossovers (Figs. 1B, C) (see also [Hunter 2015]). Crossovers are reciprocal exchanges of chromosome arms flanking the original DSB site, whereas noncrossovers do not involve such an exchange. Theoretically, HJs are symmetrical structures and one would expect a 1:1 ratio of crossovers and noncrossovers from $\mathrm{HJ}$ resolution, however, it has been proposed that during meiosis double HJs in S. cerevisiae are almost exclusively resolved into crossovers [Allers and Lichten 2001]; the mechanistic details of this bias in $\mathrm{HJ}$ resolution towards crossover are still unclear. Double HJs can also be converted into hemicatenanes through branch migration by a RecQ-type DNA helicase (HJ dissolution) (Fig. 1C) (reviewed in [Bizard and Hickson 2014]); a process which likely does not occur in Sz. pombe due to the absence of double HJs [Cromie et al. 2006], and which in S. cerevisiae does not seem to be active during meiosis [Kaur et al. 2015; Tang et al. 2015].

Conceptually, two main stages of meiotic recombination pathway decisions could be discerned: firstly, "template choice", at which it is decided whether the homologue or the sister chromatid will be the template for repair (Fig. 1A); secondly, "crossover/noncrossover decision", which occurs after the invasion of an intact chromosome by its broken homologue and the initiation of repair, and results in either a crossover or a non-crossover recombinant (Figs. 1B, C). Because homologues are not identical DNA molecules, heteroduplex DNA (a region of hybrid DNA containing one or more mismatches) may be formed in the proximity of the original DSB after repair. Subsequent mismatch correction can lead to gene conversion which is defined as the non-reciprocal transfer of genetic information from one homologue to the other. Gene conversion can be associated with both crossovers and non-crossovers. Only crossovers between homologues support the formation of chiasmata, and thus the correct segregation of chromosomes. Therefore, careful regulation of this complex process is imperative to ensure the complete repair of all DSBs and the required number of chiasmata between the correct partner chromosomes.

The ATP-fuelled capability of DNA helicases to translocate along DNA and disrupt DNA-DNA and/or DNA-protein interactions allows them to shape the meiotic recombination landscape. The regulation and modulation of meiotic recombination is achieved by DNA helicases in an intricate, often antagonistic interplay with recombinases (Rad51 and Dmc1) and their accessory factors (Rad51/Dmc1-mediators) [Lorenz et al. 2014; Brown and Bishop 2015]. Depending on the circumstances DNA helicases can act as either anti- or pro-crossover factors. At several steps in the homologous recombination pathways described above, DNA helicases are involved and may determine meiotic recombination outcome. DNA helicases are instrumental in disrupting D-loops and in branchmigrating HJs, and maybe contribute to the efficiency of DNA resection (reviewed in [Mimitou and Symington 2011]). In the following section I will discuss how different types of DNA helicases are involved in various distinct steps of meiotic recombination, and highlight questions which, despite major recent advances in the field, still remain unanswered.

\section{ScSgs1 and SpRqh1: the RecQ-type DNA helicases}

RecQ-type DNA helicases are ubiquitous; genomes of prokaryotes tend to have a single representative of this protein family, whereas most eukaryotic genomes harbour two or more paralogues (reviewed in [Ashton and Hickson 2010; Knoll and Puchta 2011]). The RecQtype helicase involved in homologous recombination in S. cerevisiae is called Sgs1, and the Sz. pombe one Rqh1. Initial characterization of sgs 1 deletion mutants in $S$. cerevisiae revealed an increase in initiation sites of synaptonemal complex formation and meiotic crossovers without affecting gene conversion frequency [Rockmill et al. 2003]. More detailed molecular biology studies by the Lichten and Hunter labs demonstrated that Sgs1 is not only a key generator of non-crossover outcomes but apparently functions as a central conductor of meiotic recombination events. Sgs1 disassembles D-loops to drive non-crossover formation by SDSA (Fig. 1A), regulates the proper formation of late recombination intermediates, and prevents the accumulation of complex and aberrant joint molecules involving multiple chromatids [Oh et al. 2007; De Muyt et al. 2012; Zakharyevich et al. 2012]. A mechanistic basis for these roles is underpinned by in vitro data showing that full-length Sgs1 is an ATPdependent $3^{\prime} \rightarrow 5^{\prime}$ 

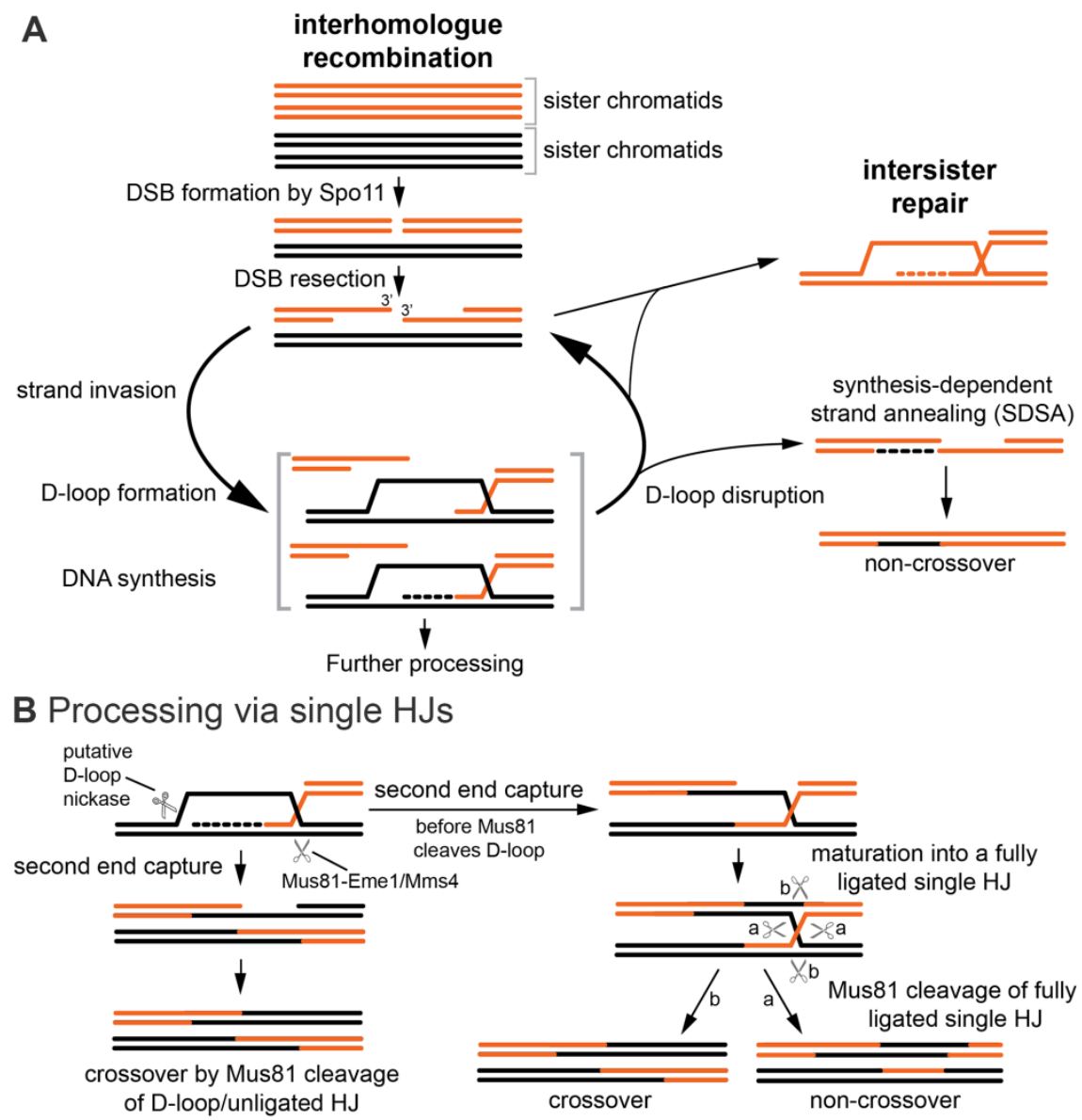

C Processing via doube HJs

second end capture

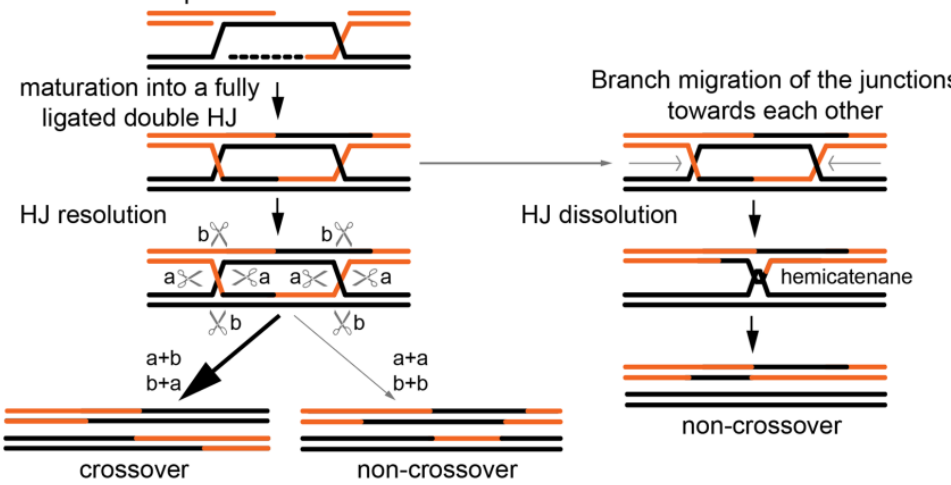

Figure 1. Schematics of meiotic recombination pathways. A homologous chromosome pair is represented by orange and black sister chromatids, for the sake of simplicity only the chromatid pair involved in recombination is depicted after the first step (DSB formation by Spo11) of the pathway(s). Outcomes of recombination are depicted with heteroduplex DNA intact, in vivo this will be restored or converted by mismatch repair. (A) Showing early steps of recombination from DSB induction to Dloop formation (the second end is shown but has not been captured yet by the D-loop). Alternative routes lead to intersister repair, and synthesis-dependent strand annealing (SDSA). SDSA exclusively results in non-crossovers. (B) Processing via single $\mathrm{HJs}$ is the predominant pathway in Sz. pombe meiosis; processing of D-loops and unligated HJs will exclusively produce crossovers, whereas processing of fully ligated $\mathrm{HJ}$ s can result in both crossovers and non-crossovers. (C) Processing via double $\mathrm{HJs}$ is the main pathway in S. cerevisiae meiosis; after the second DSB end is captured and the double HJ is fully ligated, HJ resolution by endonucleolytic cleavage can lead to both crossovers and non-crossovers, although it has been suggested that by a yet uncharacterized mechanism only crossovers are generated during meiosis in S. cerevisiae (see main text for details); this is indicated by the size and weight of the arrows leading to a particular outcome. Double HJ dissolution results in noncrossovers only, however this pathway does not seem to be active in S. cerevisiae meiosis (see main text for details). 
helicase capable of binding and dismantling a wide range of different DNA substrates, including HJs [Cejka and Kowalczykowski 2010]. As mentioned before, the branch migration activity of Sgs1 is also important for double HJ dissolution (Fig. 1C), and, similar to its mammalian orthologue BLM, can deliver noncrossovers supported by Rmi1 and Top3 (RMI1 and TOP $3 \alpha$ in mammals) via this pathway [Bizard and Hickson 2014; Wu and Hickson 2003]. In vitro, the activity of Sgs1 and BLM is vastly improved by the presence of their interaction partners Rmi1/RMI1 and Top3/TOP3 $\alpha$ [Wu et al. 2006; Cejka et al. 2010]. The double $\mathrm{HJ}$ dissolution pathway does not seem to be implemented during meiosis in $S$. cerevisiae, but unexpectedly Rmi1 is important for all meiotic functions of Sgs1 suggesting that the Sgs1-(Top3-)Rmi1 complex delivers more activities than double $\mathrm{HJ}$ dissolution [Kaur et al. 2015; Tang et al. 2015].

During DSB repair in vegetative $S$. cerevisiae cells DNA resection is initiated by the MRX (Mre11-Rad50Xrs2) complex and then continued by the exonuclease Exo1 and/or Sgs1-Top3-Rmi1 together with the endonuclease Dna2 (reviewed in [Mimitou and Symington 2011]). Intriguingly, in S. cerevisiae after initiation by the MRX complex DNA resection is apparently driven mainly by Exo1 during meiosis, with Sgs1 having only a marginal, if any, role [Zakharyevich et al. 2010; Keelagher et al. 2011]. However, at later steps of meiotic recombination during $\mathrm{HJ}$ resolution Sgs1 and Exo1 apparently collaborate to deliver wildtype levels of crossovers within the Mlh1-Mlh3 pathway [Zakharyevich et al. 2010; Zakharyevich et al. 2012]. This late role of Sgs1 also establishes that in certain situations it does work as a pro-crossover factor.

The meiotic roles of Rqh1 in Sz. pombe seem to be quite different from the ones of Sgs1 in S. cerevisiae; one of the most obvious differences is that the deletion of rqh1 causes a strong reduction of meiotic recombination frequencies [Cromie et al. 2008; Lorenz et al. 2012]. Because in rqh1 $\Delta$ mutants DSB formation is not reduced and intersister repair is not increased, it has been suggested that this reduction in meiotic recombination frequencies is caused by a lack of Rqh1mediated branch migration leading to D-loop extension (Fig. 1A), which in a wild-type situation could promote gene conversion and crossover frequency [Cromie et al. 2008]. Another contributing factor could be the involvement of RecQ-type DNA helicases in DNA resection which works in parallel to the DNA resection

Lorenz (2016) activity of Exo1 [Mimitou and Symington 2011]; consistently, an exo1s rqh1s double mutant displays lower gene conversion rates than either single mutant in meiosis [Osman et al. 2016]. On the face of this, Rqh1 looks like a straight-forward pro-crossover factor in $\mathrm{Sz}$. pombe. However, Rqh1 is required to keep meiotic gene conversion-associated crossover levels low in the absence of the Rad51/Dmc1-mediators Swi5-Sfr1, Rad55-Rad57, or Rlp1-Rdl1-Sws1, suggesting a potential anti-crossover role for Rqh1 during the "crossover/non-crossover decision" of the meiotic recombination process [Lorenz et al. 2014].

While the phenotypes of deleting SGS1 in $S$. cerevisiae and $r q h 1^{+}$in $S z$. pombe are quite different, RecQ-type DNA helicases clearly have important roles in both model yeasts, which likely reflects the capability of RecQ-type DNA helicases to interfere with decisions and processes at multiple steps within the recombination pathway (Fig. 1). Currently, it seems as if Sgs1 has an anti-recombinogenic role early-on diverting events away from becoming fully-fledged HJs between homologous chromosomes and a prorecombinogenic role later-on by supporting the Mlh1Mlh3 endonuclease. Rqh1, on the other hand, has an early pro-recombinogenic role ensuring wild-type levels of recombination events between homologues and a potential late anti-recombinogenic role in promoting non-crossover outcomes among gene conversion events. Future work will need to dissect their particular contribution at each step they are involved in and determine which interactions are required to perform the required task(s).

\section{SpFml1 and ScMph1: the FANCM-like DNA helicases}

FANCM-like DNA helicases include $S z$. pombe Fml1, $S$. cerevisiae Mph1, and archaeal Hef. These proteins primarily target DNA junctions when DNA replication is perturbed, and intermediates of homologous recombination (reviewed in [Whitby 2010; Xue et al. 2015a]). One of the main roles of Fml1 and Mph1 in homologous recombination is crossover avoidance during DSB repair in vegetative cells [Sun et al. 2008; Prakash et al. 2009]. The other FANCM-paralog found in the $S z$. pombe genome, Fml2, has no apparent role in DNA replication-associated repair or homologous recombination, even double mutant combinations with fml1s do not exacerbate the effect of a fml1s single mutant [Sun et al. 2008; Lorenz et al. 2012]. In vitro

Yeast DOI:10.1002/yea.3227 
Fml1 and Mph1 are able to dismantle a wide range of synthetic, branched DNA substrates, including those mimicking meiotic recombination intermediates such as D-loops and HJs [Sun et al. 2008; Prakash et al. 2009].

In vivo Fml1 and Mph1 function is modulated by the histone-fold proteins Mhf1 and Mhf2 [Yan et al. 2010; Bhattacharjee et al. 2013; Xue et al. 2015b]. In $S$. cerevisiae Mhf1-Mhf2 supports Mph1 only in replication-associated functions, but not in crossover avoidance [Xue et al. 2015b]. In contrast, Sz. pombe Fml1 is promoted by Mhf1-Mhf2 for all its activities [Bhattacharjee et al. 2013]. Intriguingly, in Sz. pombe Mhf1-Mhf2 (a.k.a. CENP-S and CENP-X) also have a crucial Fml1-independent role at the kinetochore; thus the loss of Mhf1 or Mhf2 function actually causes a more dramatic cellular phenotype than the deletion of $\mathrm{fml1}$ [Bhattacharjee et al. 2013].

Another Fml1/Mph1-binding protein is Dbl2/Mte1 which localizes to DSBs and is needed to recruit Fml1/Mph1 to these breaks [Yu et al. 2013; Xue et al. 2016; Yimit et al. 2016]. Biochemically Mte1 enhances Mph1 activity during replication fork regression, but negatively regulates its anti-crossover function; this basically makes Mte1 a pro-crossover factor during DSB repair [Xue et al. 2016].

One could assume, that the biochemical capability of an Mph1-Mhf1-Mhf2-Mte1 complex would predestine it to also shape the homologous recombination landscape during meiosis in $S$. cerevisiae; however this is not the case, and neither mph1 nor mte1 mutants display a noteworthy meiotic recombination phenotype [Xue et al. 2016] (Michael Lichten, pers. comm.).

In Sz. pombe on the other hand Fml1-Mhf1-Mhf2 is important for delivering a subset of non-crossover recombinants [Lorenz et al. 2012; Bhattacharjee et al. 2013], presumably by driving the SDSA pathway (Fig. 1A). Indeed, the ATPase activity and the ability to interact with Mhf1-Mhf2 are absolutely essential for Fml1's meiotic role [Lorenz et al. 2012; Bhattacharjee et al. 2013]. Fml1-Mhf1-Mhf2 seems to compete for the same recombination intermediates as the structureselective endonuclease Mus81-Eme1 [Lorenz et al. 2012], which is the sole mechanism by which crossovers are delivered in Sz. pombe meiosis (Fig. 1B) [Osman et al. 2003; Smith et al. 2003; Cromie et al. 2006]. In a wild-type situation crossover formation by Mus81-Eme1 is favoured by the Rad51/Dmc1mediators (Swi5-Sfr1, Rad55-Rad57, and Rlp1-Rdl1-

Lorenz (2016)
Sws1) which seem to curb Fml1 (and potentially Rqh1) activity on recombination intermediates [Lorenz et al. 2012; Lorenz et al. 2014]. Indeed, deleting a Rad51/Dmc1-mediator or over-expressing Fml1 reduces gene conversion-associated crossover frequency and partially rescues the low spore viability of a mus81 $\Delta$ mutant [Hyppa and Smith 2010; Lorenz et al. 2012; Lorenz et al. 2014]. Whether Dbl2 contributes to Fml1-Mhf1-Mhf2 function is currently unclear, because Dbl2 is important for localization of the UvrDtype DNA helicase Fbh1 [Polakova et al. 2016], and since the associated phenotype is upstream of Fml1's roles it masks a potential genetic interaction between Dbl2 and Fml1-Mhf1-Mhf2 (see below).

Overall, FANCM-like helicases play an important role in crossover avoidance during DSB repair in vegetative cell of both yeasts, but only in Sz. pombe does this function extend to meiotic recombination. An interesting question was raised by the discovery that Dbl2 seems to primarily support Fbh1 rather than Fml1 function, and it will be important to disrupt the physical interaction between Fml1 and Dbl2 by targeted mutations in order to understand whether $\mathrm{Dbl} 2$ is important for Fml1's meiotic role.

\section{Foh1 and Srs2: the UvrD-type DNA helicases}

UvrD-type DNA helicases are highly conserved, most organisms containing at least one representative. $S$. cerevisiae contains only Srs2, which has been shown to maintain genome stability in vegetative cells via various pathways (reviewed in [Marini and Krejci 2010]), and indeed one of Srs2's key abilities is removing the recombinase Rad51 from DNA [Krejci et al. 2003; Veaute et al. 2003]. This antirecombinase function of Srs2 is counteracted by Rad51-paralogues and mediators, such as Rad55-Rad57, the $S$. cerevisiae PCSS or Shu complex (consisting of Psy3-Csm2-Shu1-Shu2) and its Sz. pombe equivalent Rlp1-Rdl1-Sws1, which promote Rad51-dependent homologous recombination [Doe and Whitby 2004; Martín et al. 2006; Bernstein et al. 2011; Liu et al. 2011].

Its biochemical activity would make Srs2 a prime candidate for shaping meiotic recombination outcome. Intriguingly, the meiotic phenotype of an srs 2 mutant in $S$ cerevisiae is rather moderate, and it shows some, but not major, changes in gene conversion or crossover frequency [Palladino and Klein 1992; Sasanuma et al. 2013a; Hong and Kim 2013]. Importantly, the moderate Yeast DOI:10.1002/yea.3227 
reduction of spore viability seen in srs 2 mutants is apparently not related to meiotic DSB formation and repair [Palladino and Klein 1992]. Moreover, the PCSS complex which supports Rad51-dependent recombination in vegetative cells by antagonizing Srs2 [Bernstein et al. 2011], also promotes meiotic recombination, but seemingly not by modulating Srs2 activity [Sasanuma et al. 2013a; Hong and Kim 2013]. One possible explanation for Srs2 not having a major meiotic role could be that Rad51 is for the most part not an active strand exchange factor in S. cerevisiae meiosis, because its activity is blocked by the meiosis-specific Rad51-inhibitor Hed1 and primarily serves as a mediator for the meiosis-specific recombinase Dmc1 [Tsubouchi and Roeder 2006; Cloud et al. 2012; Lao et al. 2013]. However, overexpression of Srs2, which is very toxic for vegetative yeast cells [Mankouri et al. 2002], interferes with recombination in S. cerevisiae meiosis by inhibiting Rad 51 focus formation [Sasanuma et al. 2013b]. Interestingly, Dmc1 focus formation is not affected by Srs2-overexpression, which is somewhat surprising, because Hed1-deactivated Rad51 serves as a mediator for Dmc1-driven strand exchange and the absence of Rad51 causes a conspicuous reduction in Dmc1 focus intensity [Shinohara et al. 1997; Cloud et al. 2012; Lao et al. 2013]. That there is no such indirect effect on Dmc1 focus formation when Srs2 is overexpressed could be explained by Rad51 and Dmc1 being loaded by different mediators and by Srs 2 only acting on Rad51 nucleoprotein filaments once properly assembled [Sasanuma et al. 2013b].

In Sz. pombe Rad51 is an active recombinase during meiosis [Grishchuk and Kohli 2003] and there is no Hed1-orthologue. Nevertheless, no meiotic phenotype of $s r s 2 \Delta$ is apparent [Cromie et al. 2008; Lorenz et al. 2012]. This observation is perhaps explained by the presence of a second UvrD-type DNA helicase in Sz. pombe, called Fbh1 (S. cerevisiae lacks an Fbh1 orthologue] [Osman et al. 2005; Morishita et al. 2005].

Fbh1 is unique in carrying an F-box domain at its $\mathrm{N}$ terminus in addition to its C-terminal UvrD domain. F-box domain proteins interact with Skp1 and Cullin to form SCF complexes. As a class of ubiquitin E3 ligases SCF complexes play an important part in regulating protein stability by mediating substrate specificity for ubiquitin E2 enzymes (reviewed in [Hermand 2006]). Similar to Srs2, Fbh1 is capable of removing Rad51 from DNA in vitro [Tsutsui et al. 2014], and Rad51 does

Lorenz (2016) accumulate in meiotic cells in the absence of $f b h 1$ [Sun et al. 2011]. The helicase function of Fbh1 seems to be the main effector of meiotic success, since both deletion and ATPase-deactivating mutants of $f b h 1$ show a strong reduction in spore viability [Sun et al. 2011]. Indeed, helicase-dead Fbh1 is incapable of removing Rad51 from DNA in vitro [Tsutsui et al. 2014]. In vitro an SCF complex consisting of Fbh1, Skp1, Pcu1 and Rbx1 is also able to ubiquitinate Rad51, thereby marking it for degradation. However, ubiquitination of Rad51 by an Fbh1-SCF complex is blocked in the presence of the Rad51/Dmc1-mediator Swi5-Sfr1 and when Rad51 is bound to single-stranded DNA [Tsutsui et al. 2014]. In vivo, the F-box function of Fbh1 is important for controlling Rad51 protein abundance in stationary phase cells [Tsutsui et al. 2014], but mutating it has only a moderate influence on meiotic success [Sun et al. 2011]. Intriguingly, Skp1 and Fbh1 seem to work as a unit for Fbh1's helicase function [Tsutsui et al. 2014], and an skp1 mutant does mirror most meiotic defects of an $f b h 1$ deletion, such as the accumulation of Rad 51 foci [Okamoto et al. 2012].

As mentioned above, Dbl2, a factor reported to recruit Fml1 to DSBs in vegetative cells [Yu et al. 2013], apparently has a role in promoting Fbh1 focus formation during meiosis. A dbl2 $\Delta$ mutant displays very similar meiotic phenotypes to a $f b h 1 \Delta$ mutant, including severe chromosome segregation defects and an accumulation of Rad51 foci [Polakova et al. 2016]. A physical interaction between Fbh 1 and Dbl 2 could not be demonstrated, but clearly $\mathrm{Dbl} 2$ has a role in controlling Rad51 deposition on chromatin by promoting Fbh1 action [Polakova et al. 2016].

In either yeast it is still unclear whether Srs 2 modulates Rad51 loading onto DNA during meiosis in a wild-type situation, and additional experiments are needed to dissect and understand the exact contribution of Srs 2 to meiotic recombination. $S z$. pombe definitely utilizes the other UvrD-type DNA helicase Fbh1 as the main modulator of Rad51 recruitment during meiosis, and it will be interesting to learn whether Fbh1 is also able to negatively regulate Dmc1 loading.

\section{The pro-crossover factor: Mer3 DNA helicase}

The meiosis-specific DEAD/DEAH-box DNA helicase Mer3 works in a $3^{\prime} \rightarrow 5^{\prime}$ direction which seems to predestine it to extend D-loops to promote crossover formation in S. cerevisiae [Mazina et al. 2004]. Yeast DOI:10.1002/yea.3227 
Intriguingly, in the absence of Mer3 it is Sgs1 which drastically reduces crossover frequency [Jessop et al. 2006]. The Sz. pombe genome lacks a clear orthologue candidate gene of MER3.

\section{Conclusion}

It almost seems as if S. cerevisiae is less reliant on DNA helicases shaping its meiotic recombination landscape, with the RecQ-type DNA helicase Sgs1 used as the main regulator of meiotic recombination and the procrossover factor Mer3, whereas Sz. pombe uses Rqh1 (RecQ-type), Fml1 (FANCM-like), and Fbh1 (UvrD-type) DNA helicases to modulate recombination levels. These differences are not surprising considering the massive evolutionary distance between $S$. cerevisiae and $S z$. pombe; this distance strongly underpins their usefulness as comparative research models for basic cellular processes, such as meiotic recombination.

Acknowledgements I am grateful to Simon D. Brown, Anne D. Donaldson and Takashi Kubota for critically reading the manuscript.

\section{References}

Allers T, Lichten M. 2001. Differential timing and control of noncrossover and crossover recombination during meiosis. Cell, 106: 47-57.

Ashton TM, Hickson ID. 2010. Yeast as a model system to study RecQ helicase function. DNA Repair (Amst)., 9: 303-14.

Bernstein K, Reid RJD, Sunjevaric I, Demuth K, Burgess RC, Rothstein R. 2011. The Shu complex, which contains Rad51 paralogues, promotes DNA repair through inhibition of the Srs2 anti-recombinase. Mol. Biol. Cell, 22: 1599-607.

Bhattacharjee S, Osman F, Feeney L, Lorenz A, Bryer C, Whitby MC. 2013. MHF1-2/CENP-S-X performs distinct roles in centromere metabolism and genetic recombination. Open Biol., 3: 130102.

Bizard AH, Hickson ID. 2014. The dissolution of double Holliday junctions. Cold Spring Harb. Perspect. Biol., 6: a016477. http://www.ncbi.nlm.nih.gov/pubmed/24984776.

Brown MS, Bishop DK. 2015. DNA strand exchange and RecA homologs in meiosis. Cold Spring Harb. Perspect. Biol., 7: a016659.

Cejka P, Kowalczykowski SC. 2010. The full-length Saccharomyces cerevisiae Sgs1 protein is a vigorous DNA helicase that preferentially unwinds holliday junctions. J. Biol. Chem., 285: 8290-8301.

Cejka P, Plank JL, Bachrati CZ, Hickson ID, Kowalczykowski SC. 2010. Rmi1 stimulates decatenation of double Holliday junctions during dissolution by Sgs1-Top3. Nat. Struct. Mol. Biol., 17: 1377-82.

Cloud V, Chan Y-L, Grubb J, Budke B, Bishop DK. 2012. Rad51 is an accessory factor for Dmc1-mediated joint molecule formation during meiosis. Science, 337: 1222-5.

Cromie GA, Hyppa RW, Smith GR. 2008. The fission yeast Lorenz (2016)
BLM homolog Rqh1 promotes meiotic recombination. Genetics, 179: 1157-67.

Cromie GA, Hyppa RW, Taylor AF, Zakharyevich K, Hunter N, Smith GR. 2006. Single Holliday junctions are intermediates of meiotic recombination. Cell, 127: 1167-78.

De Muyt A, Jessop L, Kolar E, Sourirajan A, Chen J, Dayani Y, Lichten M. 2012. BLM helicase ortholog Sgs1 is a central regulator of meiotic recombination intermediate metabolism. Mol. Cell, 46: 43-53.

Doe CL, Whitby MC. 2004. The involvement of Srs2 in postreplication repair and homologous recombination in fission yeast. Nucleic Acids Res., 32: 1480-91.

Grishchuk AL, Kohli J. 2003. Five RecA-like proteins of Schizosaccharomyces pombe are involved in meiotic recombination. Genetics, 165: 1031-43.

Hermand D. 2006. F-box proteins: more than baits for the SCF? Cell Div., 1: 30.

Hong S, Kim KP. 2013. Shu1 promotes homolog bias of meiotic recombination in Saccharomyces cerevisiae. Mol. Cells, 36: 446-54.

Hunter N. 2015. Meiotic recombination: the essence of heredity. Cold Spring Harb. Perspect. Biol., 7: a016618.

Hyppa RW, Smith GR. 2010. Crossover invariance determined by partner choice for meiotic DNA break repair. Cell, 142: 243-55.

Jessop L, Rockmill B, Roeder GS, Lichten M. 2006. Meiotic chromosome synapsis-promoting proteins antagonize the anti-crossover activity of Sgs1. PLoS Genet., 2: e155.

Kaur H, De Muyt A, Lichten M. 2015. Top3-Rmi1 DNA singlestrand decatenase is integral to the formation and resolution of meiotic recombination intermediates. Mol. Cell, 57: 583-94.

Keelagher RE, Cotton VE, Goldman ASH, Borts RH. 2011. Separable roles for Exonuclease I in meiotic DNA double-strand break repair. DNA Repair (Amst)., 10: 126-37.

Knoll A, Puchta H. 2011. The role of DNA helicases and their interaction partners in genome stability and meiotic recombination in plants. J. Exp. Bot., 62: 1565-79.

Krejci L, Van Komen S, Li Y, Villemain J, Reddy MS, Klein H, Ellenberger T, Sung P. 2003. DNA helicase Srs2 disrupts the Rad51 presynaptic filament. Nature, $\mathbf{4 2 3}$ 305-9.

Lam I, Keeney S. 2015. Mechanism and regulation of meiotic recombination initiation. Cold Spring Harb. Perspect. Biol., 7: a016634.

Lao JP, Cloud V, Huang C-C, Grubb J, Thacker D, Lee C-Y, Dresser ME, Hunter N, Bishop DK. 2013. Meiotic crossover control by concerted action of Rad51-Dmc1 in homolog template bias and robust homeostatic regulation. PLoS Genet., 9: e1003978.

Liu J, Renault L, Veaute X, Fabre F, Stahlberg H, Heyer W-D. 2011. Rad51 paralogues Rad55-Rad57 balance the antirecombinase Srs2 in Rad51 filament formation. Nature, 479: 245-8.

Lorenz A, Mehats A, Osman F, Whitby MC. 2014. Rad51/Dmc1 paralogs and mediators oppose DNA helicases to limit hybrid DNA formation and promote crossovers during meiotic recombination. Nucleic Acids Res., 42: 13723-13735.

Lorenz A, Osman F, Sun W, Nandi S, Steinacher R, Whitby MC. 
2012. The fission yeast FANCM ortholog directs noncrossover recombination during meiosis. Science, 336: 1585-8.

Mankouri HW, Craig TJ, Morgan A. 2002. SGS1 is a multicopy suppressor of srs2: functional overlap between DNA helicases. Nucleic Acids Res., 30: 1103-13.

Marini V, Krejci L. 2010. Srs2: The “Odd-Job Man” in DNA repair. DNA Repair (Amst)., 9: 268-275.

Martín V, Chahwan C, Gao H, Blais V, Wohlschlegel J, Russell P, Yates JR, McGowan CH. 2006. Sws1 is a conserved regulator of homologous recombination in eukaryotic cells. EMBO J., 25: 2564-74.

Mazina OM, Mazin A V, Nakagawa T, Kolodner RD, Kowalczykowski SC. 2004. Saccharomyces cerevisiae Mer3 helicase stimulates 3'-5' heteroduplex extension by Rad51; implications for crossover control in meiotic recombination. Cell, 117: 47-56.

Mimitou EP, Symington LS. 2011. DNA end resectionunraveling the tail. DNA Repair (Amst)., 10: 344-8.

Morishita T, Furukawa F, Sakaguchi C, Toda T, Carr AM, Iwasaki H, Shinagawa H. 2005. Role of the Schizosaccharomyces pombe F-Box DNA helicase in processing recombination intermediates. Mol. Cell. Biol., 25: 8074-83.

Oh SD, Lao JP, Hwang PY-H, Taylor AF, Smith GR, Hunter N. 2007. BLM ortholog, Sgs1, prevents aberrant crossingover by suppressing formation of multichromatid joint molecules. Cell, 130: 259-72.

Okamoto S, Sato M, Toda T, Yamamoto M. 2012. SCF ensures meiotic chromosome segregation through a resolution of meiotic recombination intermediates. PLoS One, 7 : e30622.

Osman F, Ahn JS, Lorenz A, Whitby MC. 2016. The RecQ DNA helicase Rqh1 constrains Exonuclease 1-dependent recombination at stalled replication forks. Sci. Rep., 6: 22837.

Osman F, Dixon J, Barr AR, Whitby MC. 2005. The F-Box DNA helicase Fbh1 prevents Rhp51-dependent recombination without mediator proteins. Mol. Cell. Biol., 25: 8084-96.

Osman F, Dixon J, Doe CL, Whitby MC. 2003. Generating crossovers by resolution of nicked Holliday junctions: a role for Mus81-Eme1 in meiosis. Mol. Cell, 12: 76174.

Palladino F, Klein HL. 1992. Analysis of mitotic and meiotic defects in Saccharomyces cerevisiae SRS2 DNA helicase mutants. Genetics, 132: 23-37.

Polakova S, Molnarova L, Hyppa RW, Benko Z, Misova I, Schleiffer A, Smith GR, Gregan J. 2016. Dbl2 regulates Rad51 and DNA joint molecule metabolism to ensure proper meiotic cromosome segregation. PLoS Genet., 12: e1006102.

Prakash R Krejci L, Van Komen S, Schürer K, Kramer W, Sung P. 2009. Yeast Mph1 helicase dissociates Rad51-made D-loops: implications for crossover control in mitotic recombination. Genes Dev., 23: 67-79.

Rockmill B, Fung JC, Branda SS, Roeder GS. 2003. The Sgs1 helicase regulates chromosome synapsis and meiotic crossing over. Curr. Biol., 13: 1954-62.

Sasanuma H, Furihata Y, Shinohara M, Shinohara A. 2013b. Remodeling of the Rad51 DNA strand-exchange protein by the Srs2 helicase. Genetics, 194: 859-72.

Sasanuma H, Tawaramoto MS, Lao JP, Hosaka H, Sanda E,
Suzuki M, Yamashita E, Hunter N, Shinohara M, Nakagawa A, Shinohara A. 2013a. A new protein complex promoting the assembly of Rad51 filaments. Nat. Commun., 4: 1676.

Schwacha A, Kleckner N. 1995. Identification of double Holliday junctions as intermediates in meiotic recombination. Cell, 83: 783-91.

Schwacha A, Kleckner N. 1997. Interhomolog bias during meiotic recombination: meiotic functions promote a highly differentiated interhomolog-only pathway. Cell, 90: 1123-35.

Shinohara A, Gasior S, Ogawa T, Kleckner N, Bishop DK. 1997. Saccharomyces cerevisiae recA homologues RAD51 and $D M C 1$ have both distinct and overlapping roles in meiotic recombination. Genes to Cells, 2: 615-29.

Smith GR, Boddy MN, Shanahan P, Russell P. 2003. Fission yeast Mus81.Eme1 Holliday junction resolvase is required for meiotic crossing over but not for gene conversion. Genetics, 165: 2289-93.

Sun W, Lorenz A, Osman F, Whitby MC. 2011. A failure of meiotic chromosome segregation in a fbh1 $1 \Delta$ mutant correlates with persistent Rad51-DNA associations. Nucleic Acids Res., 39: 1718-31.

Sun W, Nandi S, Osman F, Ahn JS, Jakovleska J, Lorenz A, Whitby MC. 2008. The FANCM ortholog Fml1 promotes recombination at stalled replication forks and limits crossing over during DNA double-strand break repair. Mol. Cell, 32: 118-28.

Tang S, Wu MKY, Zhang R, Hunter N. 2015. Pervasive and essential roles of the Top3-Rmi1 decatenase orchestrate recombination and facilitate chromosome segregation in meiosis. Mol. Cell, 57: 607-21.

Tsubouchi H, Roeder GS. 2006. Budding yeast Hed1 downregulates the mitotic recombination machinery when meiotic recombination is impaired. Genes Dev., 20: 1766-75.

Tsutsui Y, Kurokawa Y, Ito K, Siddique MSP, Kawano Y, Yamao F, Iwasaki H. 2014. Multiple regulation of Rad51-mediated homologous recombination by fission yeast Fbh1. PLoS Genet., 10: e1004542.

Veaute X, Jeusset J, Soustelle C, Kowalczykowski SC, Le Cam E, Fabre F. 2003. The Srs2 helicase prevents recombination by disrupting Rad51 nucleoprotein filaments. Nature, 423: 309-12.

Whitby MC. 2010. The FANCM family of DNA helicases/translocases. DNA Repair (Amst)., 9: 224236.

Wu L et al. 2006. BLAP75/RMI1 promotes the BLMdependent dissolution of homologous recombination intermediates. Proc. Natl. Acad. Sci. U. S. A., 103: 406873.

Wu L, Hickson ID. 2003. The Bloom's syndrome helicase suppresses crossing over during homologous recombination. Nature, 426: 870-4.

Xue X, Choi K, Bonner JN, Szakal B, Chen YH, Papusha A, Saro D, Niu H, Ira G, Branzei D, Sung P, Zhao X. 2015b. Selective modulation of the functions of a conserved DNA motor by a histone fold complex. Genes Dev., 29: 1000-5.

Xue X, Papusha A, Choi K, Bonner JN, Kumar S, Niu H, Kaur H, Zheng XF, Donnianni RA, Lu L, Lichten M, Zhao X, Ira G, Sung P. 2016. Differential regulation of the anticrossover and replication fork regression activities of 
Mph1 by Mte1. Genes Dev., 30: 687-99.

Xue X, Sung P, Zhao X. 2015a. Functions and regulation of the multitasking FANCM family of DNA motor proteins. Genes Dev., 29: 1777-88.

Yan Z, Delannoy M, Ling C, Daee D, Osman F, Muniandy PA, Shen X, Oostra AB, Du H, Steltenpool J, Lin T, Schuster B, Décaillet C, Stasiak A, Stasiak AZ, Stone S, Hoatlin ME, Schindler D, Woodcock CL, Joenje H, Sen R, de Winter JP, Li L, Seidman MM, Whitby MC, Myung K, Constantinou A, Wang W. 2010. A histone-fold complex and FANCM form a conserved DNAremodeling complex to maintain genome stability. Mol. Cell, 37: 865-78.

Yimit A, Kim T, Anand RP, Meister S, Ou J, Haber JE, Zhang Z, Brown GW. 2016. MTE1 functions with MPH1 in double-strand break repair. Genetics, 203: 147-57.
Yu Y, Ren J-Y, Zhang J-M, Suo F, Fang X-F, Wu F, Du L-L. 2013. A proteome-wide visual screen identifies fission yeast proteins localizing to DNA double-strand breaks. DNA Repair (Amst)., 12: 433-43.

Zakharyevich K, Ma Y, Tang S, Hwang PY-H, Boiteux S, Hunter N. 2010. Temporally and biochemically distinct activities of Exo1 during meiosis: double-strand break resection and resolution of double Holliday junctions. Mol. Cell, 40: 1001-15.

Zakharyevich K, Tang S, Ma Y, Hunter N. 2012. Delineation of joint molecule resolution pathways in meiosis identifies a crossover-specific resolvase. Cell, 149: 334-47. 